\title{
Efficacy of coronary imaging on bifurcation intervention
}

\author{
Kensuke Takagi ${ }^{1} \cdot$ Ryoji Nagoshi $^{2}$ - Byeong-Keuk Kim ${ }^{3}$. Woong Kim ${ }^{4}$ - Yoshihisa Kinoshita ${ }^{5}$. Junya Shite ${ }^{2}$. \\ Yutaka Hikichi ${ }^{6}$. Young Bin Song ${ }^{7} \cdot$ Chang-Wook Nam $^{8}$ • Bon-Kwon Koo ${ }^{9} \cdot$ Soo-Joong Kim ${ }^{10}$. \\ Yoshinobu Murasato ${ }^{11}$ (B)
}

Received: 24 August 2020 / Accepted: 25 August 2020 / Published online: 7 September 2020

(c) The Author(s) 2020

\begin{abstract}
During the coronary bifurcation intervention procedure, imaging including intravascular ultrasound and optical coherence tomography is essential to provide precise anatomy of the lesion and morphological information. This consensus document between the Korean Bifurcation Club and the Japanese Bifurcation Club summarizes practical guidelines and current evidences on lesion assessment, device selection, procedural guidance, and the optimization of bifurcation intervention by the imaging.
\end{abstract}

Keywords Intravascular ultrasound · Optical coherence tomography $\cdot$ Coronary bifurcation · Percutaneous coronary intervention

\section{Introduction}

Percutaneous coronary intervention (PCI) for bifurcation lesions remains challenging because of a relatively low success rate, high incidence of procedural complications and inferior clinical outcomes compared to those in nonbifurcation lesions; even in the new-generation drug-eluting stent (DES) era [1]. In bifurcation lesions, coronary angiography cannot accurately visualize the carina area due to overlapping of the main vessel (MV) and side branch (SB),

Kensuke Takagi, Ryoji Nagoshi and Byeong-Keuk Kim have equally contributed on the article as first author.

Soo-Joong Kim soojoong27@daum.net

Yoshinobu Murasato y.murasato@gmail.com

1 Department of Cardiology, Ogaki Municipal Hospital, Ogaki, Japan

2 Department of Cardiology, Osaka Saiseikai Nakatsu Hospital, Osaka, Japan

3 Department of Cardiology, Yonsei Severance Hospital, Seoul, South Korea

4 Department of Cardiology, Yeungnam Medical Center, Daegu, South Korea

5 Department of Cardiovascular Medicine, Toyohashi Heart Center, Toyohashi, Japan which might limit the accurate assessment of atherosclerotic involvement. On the contrary, intravascular imaging including intravascular ultrasound (IVUS) or optical coherence tomography (OCT) is useful in guiding the PCI strategy by offering helpful pre-procedural information such as lumen and vessel dimensions, and lesion characteristics during PCI. Furthermore, Imaging-guided PCI could provide more favorable outcomes than angio-guided PCI by allowing optimal expansion and apposition of the stent as well as its appropriate landing zone. In this first consensus document between the Korean Bifurcation Club (KBC) and the Japanese Bifurcation Club (JBC), we include a scientific

6 Department of Cardiology, Saga University, Saga, Japan

7 Department of Cardiology, Samsung Medical Center, Seoul, South Korea

8 Division of Cardiology, Department of Internal Medicine, Keimyung University Dongsan Hospital, Daegu, South Korea

9 Department of Internal Medicine and Cardiovascular Center, Seoul National University Hospital, Seoul, South Korea

10 Division of Cardiology, Department of Internal Medicine, Kyung Hee University College of Medicine, Kyung Hee University Hospital, 23, Kyungheedae-ro, Dongdaemun-gu, Seoul 02447, South Korea

11 Department of Cardiology and Clinical Research Center, National Hospital Organization, Kyushu Medical Center, 1-8-1, Jigyohama, Chuo, Fukuoka 810-8563, Japan 
discussion, practical guidelines, and current evidences on debatable issues about IVUS and OCT imaging assessment of bifurcation lesions.

\section{Impact of imaging-guidance on bifurcation intervention}

There are several mechanisms responsible for treatment failure in bifurcation lesions. Stent under-expansion due to insufficient preparation of severely calcified or hard fibrous plaques in lesions is one of the most leading causes for instent restenosis (ISR). Edge dissection after oversized stenting or aggressive post-dilatation also increases the risk of ISR. Coronary angiography is not able to clearly elucidate these contributing factors for stent failure and frequently shows ambiguity during bifurcation stenting [2]. In addition, it has limitations in assessing the SB ostium, overlapping stent segments, lesion coverage, stent apposition, and wire position. However, intravascular imaging studies could provide essential information for the lesion characteristics and the dimensions of the vessel and lumen in bifurcation lesions. In addition, they could help the assessment of anatomic configuration, selection of treatment strategy, and evaluation of post-treatment results including stent expansion and apposition, which are very important in the treatment of bifurcation lesions [3, 4]. Indeed, several metaanalyses of randomized trials and observational registries showed the superiority of imaging-guided PCI in complex procedures, including left main (LM) stem and bifurcations, compared to angiography-guided PCI. According to the data from a Korean multicenter bifurcation registry, IVUS-guided PCI with DES significantly reduced the incidence of death or MI compared with angiography-guided PCI in a cohort of bifurcation lesions [5]. Basically, both pre- and post-procedural observations in MV and SB are recommended with the exception of post-procedural observation of the SB jailed by the MV stent due to the risk of distortion or fracture of the stent. In the pre-procedural observation, the following assessments facilitate optimal selection of devices and PCI strategy: (1) measurement of dimensions for lumen and vessel in MV and SB; (2) assessment of atherosclerotic plaque morphology, burden, longitudinal distribution, and negative remodeling; (3) detection of angiographically silent disease; and (4) the risk of SB compromise. In the post-procedural observation, the following assessments are required to optimize the procedure: (1) stent apposition, (2) stent expansion, (3) full lesion coverage by the stent, (4) stent edge dissection, (5) plaque prolapse inside stent, (6) SB residual stenosis and dissection, and (7) optimal guidewire (GW) recrossing before SB dilation and subsequent adequate clearance of jailing struts after SB dilation.

\section{Advantage of IVUS}

In the current guidelines, IVUS-guided PCI is recommended for complex lesions including LM, bifurcation, long lesions, and chronic total occlusions due to particular challenges in angiographic evaluation and procedural complexity [6]. The 'Impact of Intravascular Ultrasound Guidance on Outcomes of Xience Prime Stents in Long Lesions (IVUS-XPL)' trial showed the superiority of IVUS-guided PCI in 1400 patients with long coronary lesions compared with angiographyguided PCI [7]. Furthermore, the ULTIMATE Trial, which randomly assigned 1448 patients to IVUS usage, also showed that IVUS-guided DES implantation significantly improved clinical outcomes of all-comers compared with angiography guidance [8]. On meta-regression analysis, IVUS lowered the mortality rate in patients with complex lesions or acute coronary syndrome [9].

On the other hand, the usefulness of IVUS is emphasized in LM PCI, especially when complex PCI is necessary. Extensive evidence supports the use of IVUS-guided LM PCI in non-randomized studies. Park et al. demonstrated that IVUSguided PCI tended to induce lower mortality rates without demonstrating a difference in myocardial infarction or target lesion revascularization (TLR) [10]. De La Torre Hernandez et al. clarified that the use of IVUS in PCI for complex LM lesions, from the data of propensity-score matched population, could significantly reduce the risk of stent thrombosis (ST) [11]. Most recently, Andell et al. reported IVUS-guidance reduced the incidence of a combined primary endpoint of mortality, ST, and restenosis in the LM PCI registry over a period of 5 years [12]. Therefore, current guidelines recommend that IVUS-guided PCI is necessary for LM bifurcation lesions to reduce adverse events. Table 1 summarizes the usefulness of IVUS during PCI for LM and bifurcation lesions.

A few studies that used greyscale or virtual histology IVUS demonstrated that plaque accumulation and vulnerability were more frequently shown in the proximal MV than in the distal MV in a bifurcation lesion [13, 14], suggesting that plaque rupture is more likely to occur in the proximal MV than in the other segments. Indeed, the CROSS trial revealed that angiographic ISR was frequently observed at the proximal stent edge after PCI with a single stent strategy in a bifurcation lesion [15]. IVUS could provide valuable information for plaque vulnerability and burdens in the bifurcation before stenting.

\section{Advantage of OCT}

Since the introduction of the frequency-domain type, OCT has been used more frequently in the guidance of PCI due to generation of high-resolution images with high speed pull 
Table 1 Characteristics of lesions, and the procedure and clinical outcome in each publication concerning the comparison between Angio-guide and IVUS-guide in coronary bifurcation PCI

\begin{tabular}{|c|c|c|c|c|c|c|c|c|}
\hline & $\begin{array}{l}\text { Year of } \\
\text { publica- } \\
\text { tion }\end{array}$ & Lesion subset & $\begin{array}{l}\text { Number of } \\
\text { patients }\end{array}$ & $\begin{array}{l}\text { Distal } \\
\text { Bifurca- } \\
\text { tion (\%) }\end{array}$ & Study type & $\begin{array}{l}\text { ACS } \\
(\%)\end{array}$ & Follow-up & $\begin{array}{l}\text { Clinical outcome } \\
\text { IVUS- vs. Angio- } \\
\text { guide }\end{array}$ \\
\hline Park SJ et al.[10] & 2009 & LM & $145 / 145$ & 53.0 & $\begin{array}{l}\text { Propensity score } \\
\text { matched }\end{array}$ & 61.2 & 3 years & $\begin{array}{l}\text { Death } \\
4.7 \% \text { vs. } 16.0 \%\end{array}$ \\
\hline $\begin{array}{l}\text { De La Torre } \\
\text { Hernandez JM, } \\
\text { et al. [11] }\end{array}$ & 2014 & LM & $505 / 505$ & 44.2 & $\begin{array}{l}\text { Propensity score } \\
\text { matched }\end{array}$ & 60.0 & 3 years & $\begin{array}{l}\text { Cardiac death/MI/ } \\
\text { TLR } 11 \% \text { vs. } \\
19 \%\end{array}$ \\
\hline Gao XF, et al.[78] & 2014 & LM & $291 / 291$ & 86.4 & $\begin{array}{l}\text { Propensity score } \\
\text { matched }\end{array}$ & NA & 1 year & $\begin{array}{l}\text { Cardiac death/MI/ } \\
\text { TLR } \\
16.2 \% \text { vs. } 24.4 \%\end{array}$ \\
\hline Tan Q, et al.[79] & 2015 & LM & $61 / 62$ & 53.7 & $\mathrm{RCT}$ & 68.3 & 2 years & $\begin{array}{l}\text { Cardiac death/MI/ } \\
\text { TLR } \\
13.1 \% \text { vs. } 29.3 \%\end{array}$ \\
\hline $\begin{array}{l}\text { Kim SH, et al. } \\
{[80]}\end{array}$ & 2010 & $\begin{array}{l}\text { Non-LM bifurca- } \\
\text { tion }\end{array}$ & $758 / 758$ & NA & $\begin{array}{l}\text { Propensity score } \\
\text { matched }\end{array}$ & 52.0 & 4 years & $\begin{array}{l}\text { All cause of } \\
\text { mortality } \\
\text { HR } 0.31(95 \% \text { CI } \\
0.13-0.74), \\
\text { Very late stent } \\
\text { thrombosis } \\
0.4 \% \text { vs } 2.8 \%\end{array}$ \\
\hline Kim JS et al. [5] & 2011 & $\begin{array}{l}\text { Non-LM bifurca- } \\
\text { tion }\end{array}$ & $487 / 487$ & NA & $\begin{array}{l}\text { Propensity score } \\
\text { matched }\end{array}$ & 53.2 & 23.7 months & $\begin{array}{l}\text { Death or MI } \\
3.8 \% \text { vs } 7.8 \% \\
\text { HR } 0.44,95 \% \text { CI } \\
0.12-0.96\end{array}$ \\
\hline $\begin{array}{l}\text { Chen SL et al. } \\
\text { [81] }\end{array}$ & 2013 & 2-stent & $123 / 123$ & NA & $\begin{array}{l}\text { Propensity score } \\
\text { matched }\end{array}$ & 87.3 & 1 year & $\begin{array}{l}\text { Stent thrombosis } \\
0 \% \text { vs } 4.9 \% \\
\text { ST-elevation MI } \\
2.4 \% \text { vs. } 9.8 \%\end{array}$ \\
\hline
\end{tabular}

$\mathrm{LM}=$ left main, $\mathrm{RCT}=$ randomized control trial, $\mathrm{MI}=$ Myocardial Infarction. $\mathrm{TLR}=$ Target Lesion Revascularization, $\mathrm{NA}=$ not available

back and convenient usability. In the latest guidelines of the European Society of Cardiology and European Association for Cardio-Thoracic Surgery, OCT as well as IVUS are recommended for procedural optimization as class IIa [16]. For OCT image acquisition, vessel flushing is necessary to remove blood and low-molecular-weight dextran is available as subsidies with contrast medium for frequent observations required in the bifurcation PCI $[17,18]$. The OCT can provide a clear visualization of coronary plaques and accurate measurement of reference lumen diameter and lesion length $[19,20]$. In the clinical study OPUS-CLASS, IVUS presented a larger minimal lumen diameter and area than OCT by $9 \%$ and $10 \%$, respectively [19]. In comparing between OCT and IVUS measurements in the phantom model, OCT was equal to the actual lumen area of the model, while IVUS overestimated and was less reproducible [19]. Hence, these characteristics are useful to decide stent size, length, landing zone, balloon size and necessity of distal protection [21]. For example, OCT can characterize the plaque components such as lipid rich or calcified plaque more clearly and we can avoid the area which contains vulnerable plaques for stent landing zone [22]. In addition, a current angio-coregistration system which reflects the location of the OCT camera on the coronary angiogram is helpful to realize stent landing zone and minimize geographic miss. After MV stenting, OCT is useful for the assessment of stent expansion, apposition, in-stent tissue protrusion, vessel dissection, GW recrossing position, and stent deformation. For the effective proximal optimization technique (POT) that facilitates optimal GW recrossing with the wide opening of the jailed struts in the SB ostium, OCT can provide useful information concerning balloon size, the length between carina and stent proximal edge, and accurate position of the balloon distal marker on the carina [23, 24]. Previous bench tests revealed that GW recrossing to the distal cell adjacent to the carina, led to wide SB opening with less malapposition after kissing balloon inflation (KBI); and GW recrossing to more proximal cell introduced protrusion of the jailed struts into the MV [24]. Clear visualization of the $\mathrm{GW}$ and stent strut in the three dimensional (3D) OCT image has a great impact on an accurate assessment of optimal GW recrossing which reduces stent malapposition significantly $(9.5 \%$ vs $42.3 \%$ in the angiography-guided group, $p<0.0001$ ) [25]. Initial angiography-guidance failed in GW recrossing to the optimal cell 
in $33-35 \%$ while OCT guidance improved the success rate up to $90-100 \%$ [26-28]. In the 3D-OCT bifurcation registry, 3D-OCT guidance allowed significantly better attainment of optimal distal GW recrossing without increasing contrast dye volume and operation time, compared with conventional 2D-OCT guidance $[27,28]$.

\section{Comparison of OCT vs. IVUS}

There are a few studies comparing OCT and IVUS as guides for PCI (Table 2) [29-31]. The OPINION study, which included bifurcation lesions in $38 \%$ of cases, revealed no statistical difference in target vessel failure between IVUS and OCT guidance [30]. Since OCT provides higher resolution images, it is useful to detect stent failure and to optimize the procedure. Unfortunately, the drawbacks of OCT were limited penetrating depth and necessity of blood clearance for the image. Hence, LM bifurcation lesion has been considered unsuitable for the OCT guidance due to more artifacts included, however, the OCT image quality in LM bifurcation was not inferior to those in non-LM bifurcation in recent studies [27, 28]. 3D- OCT guidance provided significantly less incomplete strut apposition than 2D- OCT guidance, after LM bifurcation stenting, followed by KBI with the assessment of GW recrossing point $(18.7 \pm 12.8 \%$ vs $10.3 \pm 8.9 \% ; P=0.014)$; which was not statistically significant in whole bifurcation cases $(14.5 \pm 13.6 \%$ vs $10.0 \pm 9.0 \% ; P=0.077)$ [28]. Another advantage of OCT guidance is a clear visualization of the calcium border and accurate measurement of calcium thickness that are useful for adequate lesion preparation using a rotablator in calcified lesions [32]. Calcified plaques with $<0.67 \mathrm{~mm}$ thickness were able to be dilated with cracks by balloon inflation [32]. Generally, IVUS guidance is more suitable for larger vessels and aorta-ostial lesions due to limitations of complete vessel flushing or adequate assessment in OCT guidance.

\section{Role of IVUS and OCT as predictors of TLR/ restenosis}

In general, stent under-expansion is established as a major predictor of stent failure $[33,34]$. A threshold of absolute minimum stent cross-sectional area (MSA) in IVUS analysis can be used to prevent target vessel failure involving TLR and ST. The cut-off value for MSA is $4.0-5.7 \mathrm{~mm}^{2}$ in PCI using first-generation DES [35-37]. Focusing on LM lesions, Kang SJ et al. reported that the MSA cutoffs used to predict restenosis on a segmental basis were $5.0 \mathrm{~mm}^{2}$ (ostial left circumflex artery, LCx), $6.3 \mathrm{~mm}^{2}$ (ostial left anterior descending artery, LAD), $7.2 \mathrm{~mm}^{2}$ (distal LM), and $8.2 \mathrm{~mm}^{2}$ (proximal LM) in IVUS analysis [38]. As shown in Fig. 1, stent under-expansion in LCX ostium and remaining metallic carina after 2-stenting in LM is likely to generate restenosis and IVUS-guided stent expansion was therefore useful. In previous randomized trials, IVUS criteria for optimal stent expansion were defined as a MSA greater than or equal to the distal reference, that is, a MSA $>80$ to $90 \%$ of the average reference. Importantly,

Table 2 Clinical studies comparing OCT- and IVUS-guided PCI without excluding bifurcation lesions

\begin{tabular}{|c|c|c|c|c|c|}
\hline $\begin{array}{l}\text { Study name } \\
\text { Published year }\end{array}$ & Study design & Number of Pts & Bifurcation (\%) & Primary Endpoint & Results \\
\hline $\begin{array}{l}\text { ILUMIEN II } \\
2015 \text { [31] }\end{array}$ & $\begin{array}{l}\text { Post hoc matched-paired } \\
\text { analysis of ILUMIEN I } \\
\text { and ADAPT-DES }\end{array}$ & $\begin{array}{l}\text { Matched groupsOCT: } \\
286 \\
\text { IVUS: } 286\end{array}$ & $\begin{array}{l}\text { OCT: } 97(33.9) \\
\text { IVUS: } 93(32.5)\end{array}$ & $\begin{array}{l}\text { Stent expansion (\%) } \\
\text { (MSA / mean lumen } \\
\text { area) }\end{array}$ & $\begin{array}{l}\text { OCT: } 72.8 \%[63.3-81.3] \\
\text { IVUS: } 70.6 \%[62.3-78.8] \\
\text { (median }[\mathrm{IQR}], p=0.29 \text { ) }\end{array}$ \\
\hline $\begin{array}{l}\text { ILUMIEN III } \\
2016 \text { [29] }\end{array}$ & $\begin{array}{l}\text { Randomized, controlled } \\
\text { multi-center study }\end{array}$ & $\begin{array}{l}\text { OCT: } 158 \\
\text { IVUS: } 146 \\
\text { Angiography: } 146\end{array}$ & Unknown & MSA measured by OCT & $\begin{array}{l}\text { OCT: } 5.79[4.54-7.34) \\
\text { mm }{ }^{2} \\
\text { IVUS: } 5.89[4.67-7.80) \\
\text { mm }^{2} \\
\text { Angiography } 5.49 \\
\text { [4.39-6.59] mm }{ }^{2} \\
\text { OCT vs. IVUS: non-infe- } \\
\text { rior }(p=0.001), \text { but not } \\
\text { superior }(p=0.42)\end{array}$ \\
\hline $\begin{array}{l}\text { OPINION } \\
2017[30]\end{array}$ & $\begin{array}{l}\text { Randomized, controlled } \\
\text { multi-center study }\end{array}$ & $\begin{array}{l}\text { OFDI: } 414 \\
\text { IVUS: } 415\end{array}$ & $\begin{array}{l}\text { OFDI: } 154 \text { (37.4) } \\
\text { IVUS: } 157(38.8)\end{array}$ & $\begin{array}{l}\text { TVF (Composite } \\
\text { of cardiac death, } \\
\text { target-vessel related } \\
\text { myocardial infarction } \\
\text { and ischemia-driven } \\
\text { target vessel revascu- } \\
\text { larization) }\end{array}$ & $\begin{array}{l}\text { OFDI: } 21(5.2 \%) \\
\text { IVUS: } 19(4.9 \%) \\
\text { OFDI vs. IVUS: non- } \\
\text { inferior }(P=0.042)\end{array}$ \\
\hline
\end{tabular}


Table 3 OCT studies identifying predictors of side branch compromise

\begin{tabular}{|c|c|c|c|}
\hline $\begin{array}{l}\text { Author } \\
\text { Published year }\end{array}$ & $\begin{array}{l}\text { Number of lesions / } \\
\text { complications }\end{array}$ & Results (SB compromise vs. non-compromise) & Predictors \\
\hline $\begin{array}{l}\text { Watanabe et al } \\
2014 \text { [72] }\end{array}$ & $52 / 22$ & $\begin{array}{l}\text { Frequent eccentric plaque distribution opposite to SB: } 77.3 \% \text { vs. } \\
16.7 \% \\
\text { Smaller CT (carina tip) angle: } 29.58^{\circ} \text { vs. } 65.08^{\circ} \\
\text { Shorter BP-CT length (length between proximal branching point } \\
\text { to CT): } 1.20 \mathrm{~mm} \text { vs. } 2.25 \mathrm{~mm}\end{array}$ & $\begin{array}{l}\text { CT angle }<50^{\circ} \\
\text { BP-CT length }<1.70 \mathrm{~mm}\end{array}$ \\
\hline $\begin{array}{l}\text { Fujino et al } \\
2014[71]\end{array}$ & $75 / 31$ & $\begin{array}{l}\text { Smaller bifurcation angle: } 48.55 \pm 20.26^{\circ} \text { vs. } 65.58 \pm 33.98^{\circ} \\
\text { Greater } \% \text { DS of SB in pre- and post-PCI } \\
\text { More calcified plaque }\end{array}$ & $\begin{array}{l}\text { Bifurcation angle }<70^{\circ} \\
\text { Pre-PCI, SB \% DS } \\
\text { Calcified plaque in the MV }\end{array}$ \\
\hline $\begin{array}{l}\text { Kini et al } \\
2017[82]\end{array}$ & $30 / 10$ & $\begin{array}{l}\text { Frequent lipid rich plaques (lipid arc }>90 \% \text { ): } 100 \% \text { vs. } 64 \% \\
\text { Greater maximal lipid arc: } 257^{\circ} \text { vs. } 132^{\circ} \\
\text { Lipid rip-rich plaque located contralateral to the SB: Proximal } \\
\text { MV; } 50 \% \text { vs. } 5 \% \text {, Distal MV; } 30 \% \text { vs. } 10 \%\end{array}$ & $\begin{array}{l}\text { Maximal lipid arc } \\
\text { Lipid rich plaque contralat- } \\
\text { eral to SB ostium }\end{array}$ \\
\hline
\end{tabular}

SB: Side branch, MV: Main vessel, \%DS: \% diameter stenosis

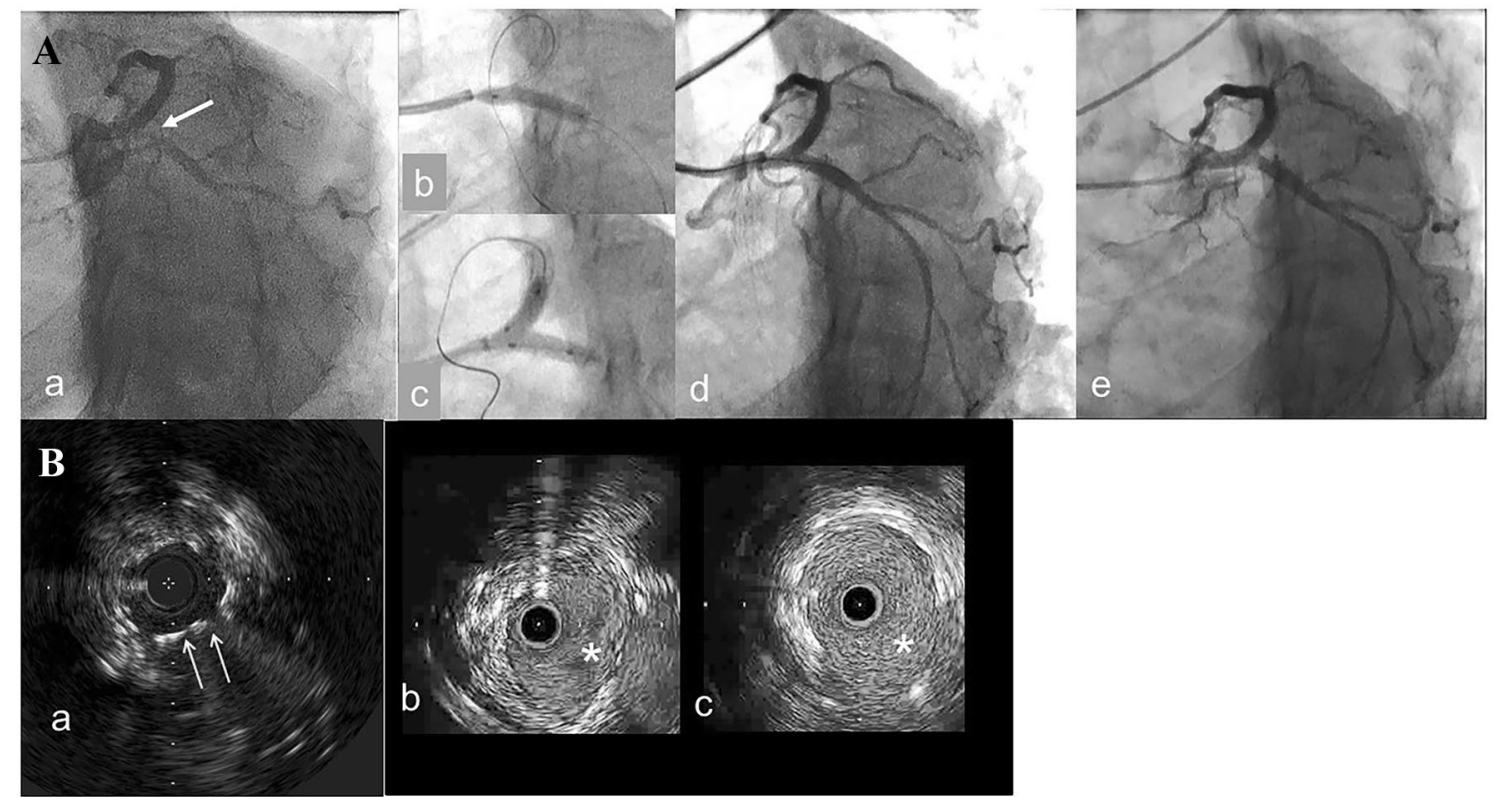

Fig. 1 A representative case of IVUS-guided treatment for the instent restenosis (ISR) after modified T-stenting in left main (LM) bifurcation performed 9 months before. Coronary angiography (CAG) showed tight ISR (A-a) and IVUS showed stent under expansion with minimal stent area (MSA) of $3.6 \mathrm{~mm} 2$ in left circumflex (LCX) ostium as well as remained metallic carina (B-a, arrows). After implantation of a $3.5 / 18 \mathrm{~mm}$ zotarolimus-eluting stent crossing over left anterior descending artery (LAD) (A-b), kissing balloon

approximately one-third of patients did not achieve the predefined criteria of stent expansion in selected randomized trials of imaging-guided PCI [6]. In the IVUSXPL trial, optimization was achieved in half of the lesions, which showed better clinical outcomes than those in which the criteria were not achieved [7]. Edge restenosis was predicted by residual plaque burden of $>51.6 \%$ to $54.5 \%$ in the DES-stented segment $[39,40]$. Therefore, the benefits inflation using two $3.5 \mathrm{~mm}$ non-compliant balloons was performed at 16 atm (A-c), which resulted in no angiographic stenosis (A-d). In the IVUS observation, both LAD (B-b) and LCX (B-c) were well expanded with MSA of $8.2 \mathrm{~mm} 2$ and $10.5 \mathrm{~mm} 2$, respectively, which led to a marked reduction of the metallic carina (asterisks). CAG at 1 -year follow-up demonstrated no significant restenosis in the treated site

of IVUS-guided PCI are gaining sufficient MSA and accurate setting of stenting zone.

The automatic measuring function in OCT is helpful to detect stent under-expansion immediately, which is established as a major predictor of stent failure [6]. Tapering from proximal to distal MV should be considered in assessing stent expansion in the bifurcation lesion. However, most of the imaging studies focused on the association between 
MSA and clinical events [34-38, 41]. The current version of OCT equips novel volumetric stent expansion analysis- referring to vessel tapering based on the $\mathrm{H}-\mathrm{K}$ equation $\left(\mathrm{Dmo}^{7 / 3}=\mathrm{Dmb}^{7 / 3}+\mathrm{Dsb}^{7 / 3}\right.$, where Dmo, Dmb and Dsb were the diameter of mother vessel, main branch and SB, respectively) $[42,43]$. With this method, the expansion index is calculated as the ratio of actual lumen area to the ideal lumen, and the minimum expansion index (MEI), demonstrated a strong correlation with device-oriented cardiac events with a cut-off value of $<73.3 \%$ [43]. This novel tapered vessel algorithm is applicable for device selection and optimization according to the vascular branching law in bifurcation stenting.

In bifurcation lesions, the predictors of TLR are more complicated because there are numerous complex bifurcation techniques and the presence and severity of SB leads to more complicated interpretations. Three mechanisms for restenosis were noted. First, ISR was caused by stent underexpansion due to insufficient preparation of severely calcified or hard fibrous plaque lesions. POT using an appropriate balloon size by intravascular imaging could more optimally and symmetrically dilate stent struts and prevent low shear stress generation, thus resulting in decreased ISR [38, 44, 45]. Second, edge dissection after oversized stenting or aggressive post-dilatation increases the risk of restenosis. Intravascular imaging-guidance could offer accurate lesion assessment and appropriate selection of device size which can prevent this kind of complication. Third, although the majority of patients with LM lesions had bi-directional plaque extension into the ostial LM and proximal LAD on IVUS observation [46], mild to moderate lesions in the proximal LM are likely to be ignored for stent coverage, resulting in proximal edge restenosis [47]. Therefore, intravascular imaging which can detect hidden, unclear plaque extension in angiography could help decide the stent landing zone. In fact, Takagi et al. showed the efficacy of the combination of POT and full-coverage ostial LM on the reduction of ISR in the ostial LM compared to propensity score-adjusted group that was not treated with this strategy [HR, 0.34 (95\% CI, $0.15-0.76), p=0.008]$ [48].

An additional two-stent strategy is necessary in 3-47\% of cases after provisional stenting [49-51]. SB dissection and bail-out two-stent deployment occurred in $10.5 \%$ and $5.6 \%$ after KBI, respectively, even when dedicated IVUS-guided KBI was performed [52], supporting the usefulness of imaging-guidance to avoid unnecessary bailout two-stenting. Large edge dissections (more than medial layer) detected by IVUS are reportedly associated with early ST [53].

The KBI is crucial in the two-stent strategy. However, in true bifurcation lesions treated with provisional single stenting, the impact of KBI remains controversial according to previous studies [54-56]. Although KBI has certain benefits of stent expansion, modifying carina shift, restoring stent shape, compressing plaque at the ostial SB, and apposing struts to the proximal $\mathrm{MV}$, there are potential concerns regarding the unfavorable impacts of KBI such as vessel dissection, asymmetric expansion, and deterioration of rheological stress due to overdilation and subsequent elliptical deformation [23, 57-59]. In the J-REVERSE trial, the KBI group obtained a greater luminal volume in the proximal MV and demonstrated less binary SB restenosis $(9.7 \%$ vs. $21.0 \%, p=0.0004)$, which was beneficial for both true and non-true bifurcation lesions. They emphasized that accurate assessment of the bifurcated vessel in terms of vessel size, plaque and intima by IVUS lead to optimal KBI treatment without increasing MV events [52]. In addition, KBI reduced proximal-segment luminal narrowing due to homogeneous neointimal distribution and fewer jailed struts at 9-month follow-up [60]. This could be partly explained by optimal balloon size guided by intravascular imaging for KBI.

\section{Role of IVUS and OCT at predicting SB compromise}

Diameter stenosis at the SB ostium and smaller carina angles is associated with SB compromise after MV stenting [61-63]. Furukawa et al. reported that IVUS could identify the presence of plaques truly involved in SB ostium that were not detected by angiography, which was associated with the SB occlusion [64]. In the bench test, Vassilev and Gil demonstrated that carina shift is a major mechanism of SB occlusion after stent crossover and that the diameter of MV at distal site of the junction and percent diameter stenosis of SB ostium are correlated with acute SB occlusion just after stent implantation from angiography [65]. On the other hand, recent IVUS analyses found that the main cause of SB compromise is a mixture of carina shift driven by distal MV lumen expansion and plaque shift [66, 67], which is not completely rectified by KBI. In the IVUS sub-study of the J-REVERSE registry, carina shift was more frequently found in cases with SB ostial residual stenosis after KBI than in those without it (37\% vs. 11\%) [66]. Independent predictors for SB residual stenosis in the pre-procedural IVUS observation were negative-remodeling at distal MV, plaque -burden at distal MV, and plaque-burden at SB ostium [66]. In addition, another IVUS study demonstrated that SB plaque burden was asymmetric and likely to present at the opposite side of flow-divider with low shear stress and SB negative remodeling was frequently encountered in the complex bifurcation lesion with extended SB lesion [68]. Therefore, IVUS, not angiography, is more helpful in precise decision making for bifurcation PCI strategy.

In cases of LM distal bifurcation, careful IVUS imaging usually showed continuous plaques from the LM into the proximal left anterior descending artery (LAD) was seen in 
$90 \%$ and from the LM into the LCx in $66 \%$, with the disease from the LM into both the LAD and LCx in 62\% [46]. Of these LM bifurcations, the patients who have a "vulnerable" carina-the eyebrow sign [69] or significant calcium [70] identified by IVUS longitudinal reconstruction, are at particular risk of adverse carina shifts towards the LCx.

The pre-procedural OCT findings are also helpful to predict SB compromise after cross-over stenting as shown in Table 3. More plaque burden, particularly calcified plaque in the MV opposite to the SB orifice [71], narrower carina tip angle and shorter length between proximal branching point to carina tip are predictors of carina shift [72]. The parallel type in which the proximal course of SB is concealed behind carina in the $3 \mathrm{D}$ perpendicular image of the SB is more likely to be associated with carina shift than the perpendicular type in which proximal SB is visualized over the carina [73].

\section{Efficacy of 3D-OCT on optimal bifurcation stenting (Table 4 and Fig. 2)}

The assessment of stent configuration over SB orifice and GW recrossing position with 3D OCT imaging before KBI provides important information to achieve optimal bifurcation stenting (Fig. 2a). Appropriate POT enlarges the distal site of jailed struts, which increases the likelihood of optimal distal wiring (Fig. 2b) [23]. In the 3D-OCT Registry, 3D-OCT guidance allowed significantly better attainment of optimal distal GW recrossing without an increase of contrast dye volume and operation time compared with the conventional 2D-OCT guidance despite of more performance of GW recrossing [27, 28]. Stent configuration was classified into two patterns. One is link-free carina type, which has no link connection on the carina, and the other is link-connecting carina type, in which the link connection is located between the carina and proximal stent strut (Fig. 2c) [27, 28, 74]. Distal GW recrossing led to better stent apposition to the lateral wall after KBI in the Link-free carina type, while in the Link-connecting type, there was no difference in stent apposition regardless of $\mathrm{GW}$ recrossing position [27, 28]. In addition, KBI with distal GW recrossing in the Link-connecting type has a potential risk of stent deformation [75]. Efficacy of KBI after cross-over stenting has been controversial because some randomized studies indicated neutral or adverse effects of KBI on clinical outcome compared to non-KBI procedures, which left jailed struts over the SB orifice $[15,56]$. However, the fact that the stent struts at ostial LCx after LM cross-over stenting impacted the narrowing of the ostial area at follow-up OCT study [76], and main pathological predictors for LM stent failure are malapposition and struts crossing an ostial LCx [77] supported the importance of the reduction of stent struts jailing the SB. The 3D- OCT imaging facilitates the achievement of

Table 4 Clinical studies on bifurcation PCI under 3D-OCT assessment

\begin{tabular}{|c|c|c|c|}
\hline $\begin{array}{l}\text { Author } \\
\text { Published year }\end{array}$ & Study design & Enrolled cases & Results \\
\hline $\begin{array}{l}\text { Okamura et al } \\
2014[26]\end{array}$ & Retrospective, single-center study & 22 & $\begin{array}{l}\text { Accurate assessment of GW recrossing by } 3 \mathrm{D}-\mathrm{OCT}: 18 / 22(81.8 \%) \\
\text { Stent configuration over SB orifice: free carina (FC) type; no link at carina } \\
(\mathrm{n}=7) \text { vs link-connecting (LC) type; the existence of link at carina }(\mathrm{n}=6) \\
\% \text { ISA after KBI: FC } 0.7 \pm 0.9 \% \text { vs. LC } 12.2 \pm 6.5 \%\end{array}$ \\
\hline $\begin{array}{l}\text { Okamura et al } \\
2018 \text { [27] }\end{array}$ & Prospective, multi-center study & 105 & $\begin{array}{l}\text { Distal cell GW recrossing under OCT guidance: } 83 \% \\
\text { \%ISA: distal GW recrossing } 6.3 \pm 6.0 \% \text { vs. proximal } 17.1 \pm 10.1 \% \\
\text { FC type with distal recrossing (LFD group, } \mathrm{n}=54 \text { ) vs. the other cases (non- } \\
\text { LFD group, } \mathrm{n}=51 \text { ) } \\
\text { \%ISA: } 6.7 \pm 5.9 \% \text { vs. } 17.0 \pm 10.5 \% \\
\text { SB restenosis at follow-up: } 8.3 \% \text { vs. } 20.5 \%, \mathrm{p}=0.1254\end{array}$ \\
\hline $\begin{array}{l}\text { Nagoshi et al } \\
2018 \text { [28] }\end{array}$ & Retrospective, multi-center study & 150 & $\begin{array}{l}\text { Distal cell GW recrossing: } 2 \mathrm{D}-\mathrm{OCT} \text { guidance } 75.6 \% \text { vs. 3D-OCT guidance } \\
\text { 91.7\% } \\
\text { \%ISA: } 2 \text {-D OCT vs. } 3 \text {-D OCT } \\
\text { SB: } 14.5 \pm 13.6 \% \text { vs } 10.0 \pm 9.0 \% \\
\text { Left main bifurcation: } 18.7 \pm 12.8 \% \text { vs } 10.3 \pm 8.9 \% \\
\text { Independent contributors to ISA: Link-connecting type, distal GW recross- } \\
\text { ing, age }\end{array}$ \\
\hline $\begin{array}{l}\text { Kume et al } \\
2018 \text { [83] }\end{array}$ & Observational, single-center study & 29 & $\begin{array}{l}\text { FC type }(n=18) \text { vs. } L C \text { type }(n=11) \\
\text { SB orifice obstruction by neointima at } 18 \text {-month follow-up: } 9.5 \pm 22.1 \% \text { vs. } \\
26.8 \pm 21.9 \%\end{array}$ \\
\hline $\begin{array}{l}\text { Fujimura et al } \\
2018 \text { [84] }\end{array}$ & Retrospective, single-center study & 37 & $\begin{array}{l}\text { Main vessel stenting followed by KBI, follow-up OCT at } 6-12 \text { month } \\
\text { LFD vs. non-LFD } \\
\text { SB ostial area gain: }+0.43 \mathrm{~mm}^{2} \text { vs. }-0.65 \mathrm{~mm}^{2},+9.47 \% \text { vs. }-13.77 \%\end{array}$ \\
\hline
\end{tabular}

$O C T$ optical coherence tomography, $G W$ guide wire, $K B I$ kissing balloon inflation, $I S A$ incomplete stent apposition, $S B$ side branch 
A

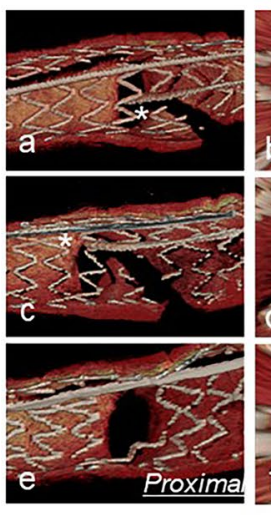

B

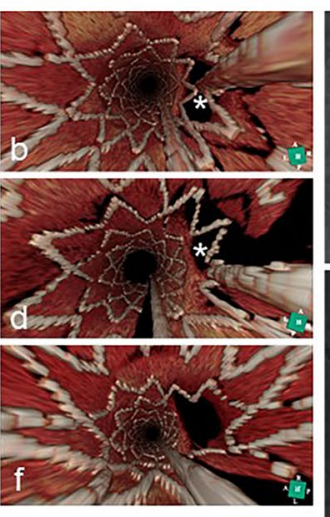

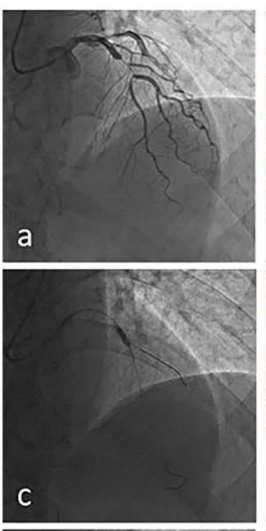

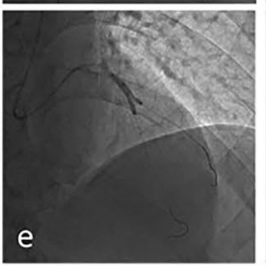

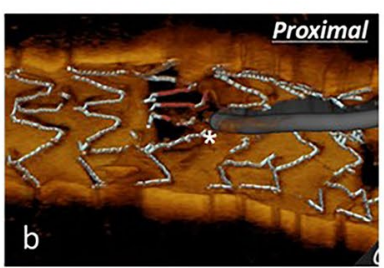
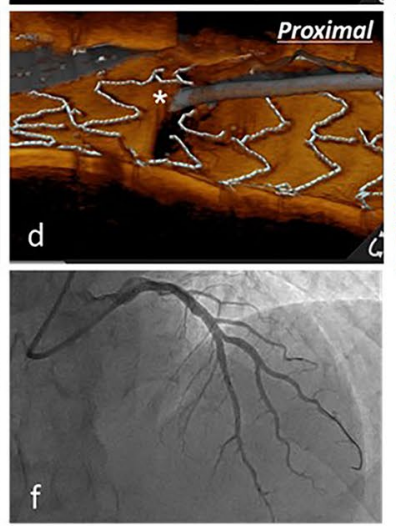

C
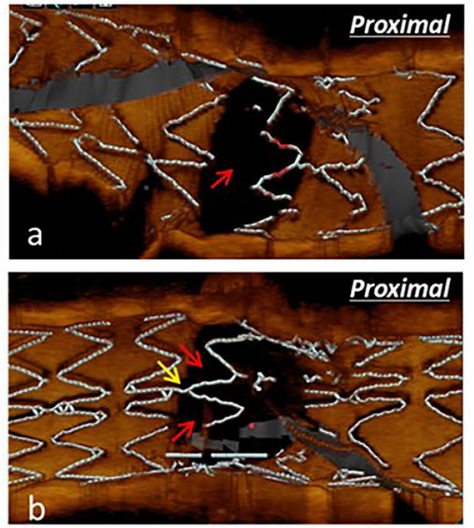

Fig. 2 A PCI for Medina $(1,1,0)$ lesion in the left anterior descending artery (LAD) - diagonal branch (Dx) bifurcation under OCT guidance. a Pre-procedural angiography. b 3-D OCT image after guidewire $(\mathrm{GW})$ recrossing toward Dx following stent implantation, which demonstrated the proximal cell GW recrossing. c POT with $3.5 \times 8 \mathrm{~mm}$ balloon. $\mathrm{d}$ 3-D OCT image after proximal optimization (POT) and the second GW recrossing toward Dx, which showed the GW recrossing into the distal cell which was enlarged by POT. e

complete removal of jailed struts and fully apposed struts in the bifurcation segment, which may lead to improvement of clinical outcome of the KBI compared to 2D imaging or angiography guidance. Such perspective is more important in two-stent strategy, which requires KBI with high pressure. The 3D-OCT guidance is also effective for optimal GW recrossing both after the first and second stent deployment in the two-stenting technique, which has the potential of improving the clinical outcome at long-term follow-up. The 3D-OCT guided PCI by non-expertise operators still has the following limitations: 1) Less identification of internal elastic lamina in severely diseased vessels is likely to lead to smaller device selection compared to IVUS [30], 2) More usage of contrast medium for vessel flushing has a possible risk of worsening renal function, and 3) Incomplete blood flushing in large vessels or shadow of the GW in 3D images is prone to inaccurate assessment of stent malapposition or GW recrossing position.

\section{Recommended procedure}

\section{i. IVUS-guided bifurcation PCI.}

Kissing balloon inflation with $2.75 \mathrm{~mm}$ and $2.5 \mathrm{~mm}$ balloons in LAD and Dx respectively. f Final angiogram. B Two patterns of stent configuration over side branch orifice. a Link-free carina type, no linkconnection is presented between carina and proximal adjacent struts (red arrow). b Link-connecting carina type, link-connection is located between carina and proximal adjacent strut (yellow arrow), which results that distal SB orifice is divided into 2 spaces by the link (red arrows)

As shown in Figs. 3 and 4, pre-procedural observation in both MV and SB is recommended for accurate assessment of lesion morphology, lumen dimension, device selection, as well as a landing zone and decision for SB treatment. If lesion preparation is necessary, pre-dilation by non-compliant or scoring balloon, rotablation or distal protection should be considered. Stent implantation according to the distal MV reference is followed by the POT with a short balloon dedicated by the IVUS. In cases of significant jailing struts in the SB ostium with a size of $\geqq 2.5 \mathrm{~mm}$, distal GW recrossing is attempted. Final KBI or simple SB dilation follows optimal GW recrossing and POT is performed as correction of stent deformation. When post-procedural failure is found in IVUS, such as stent under-expansion, malapposition, deformation, and edge dissection or residual stenosis, optimization of the procedure should be added and reassessed by the IVUS after the procedure.

\section{ii. OCT-guided bifurcation PCI}

As shown in Figs. 3 and 5, the recommended OCT-guided bifurcation PCI is basically similar to IVUS-guided PCI, whereas the assessment of GW recrossing point, link-connection and jailing struts on the SB ostium using 3D images 
Fig. 3 Flow of imaging-guided provisional bifurcation stenting. GW: guide wire, MV: main vessel, POT: proximal optimization technique, SB: side branch, FKBI: final kissing balloon inflation

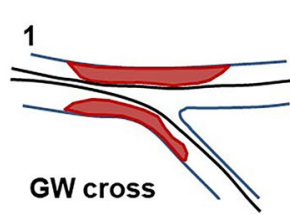

4

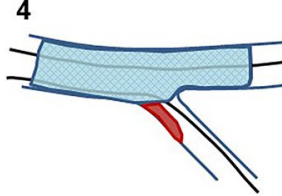

Imaging

- Lesion characteristics (lipid, fibrous, calcification, plaque burden, rupture)

- MV: stent size, length, landing zone, POT balloon size

SB: necessity of treatment balloon/stent size, landing zone

Imaging

- Stent underexpansion

malapposition, deformation

- Edge dissection, residual stenosis

- SB jailing struts
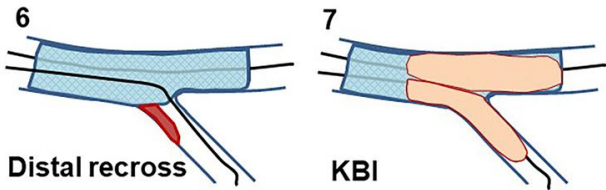

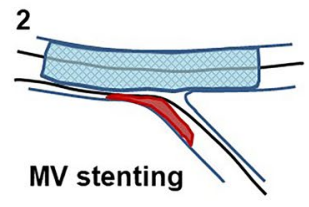

3

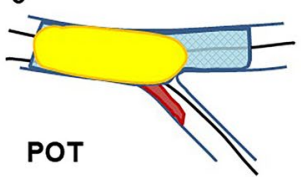

5

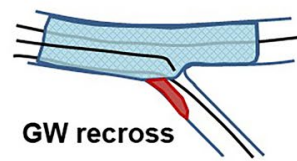

3D OCT

- GW recrossing poin

- Stent link location

- Jailing strut at SB ostium

8

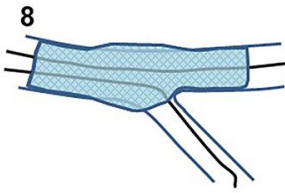

Imaging

Post dilation (higher pressure,

larger size)

- FKBI, Re-POT

- Additional stenting (SB, MV)

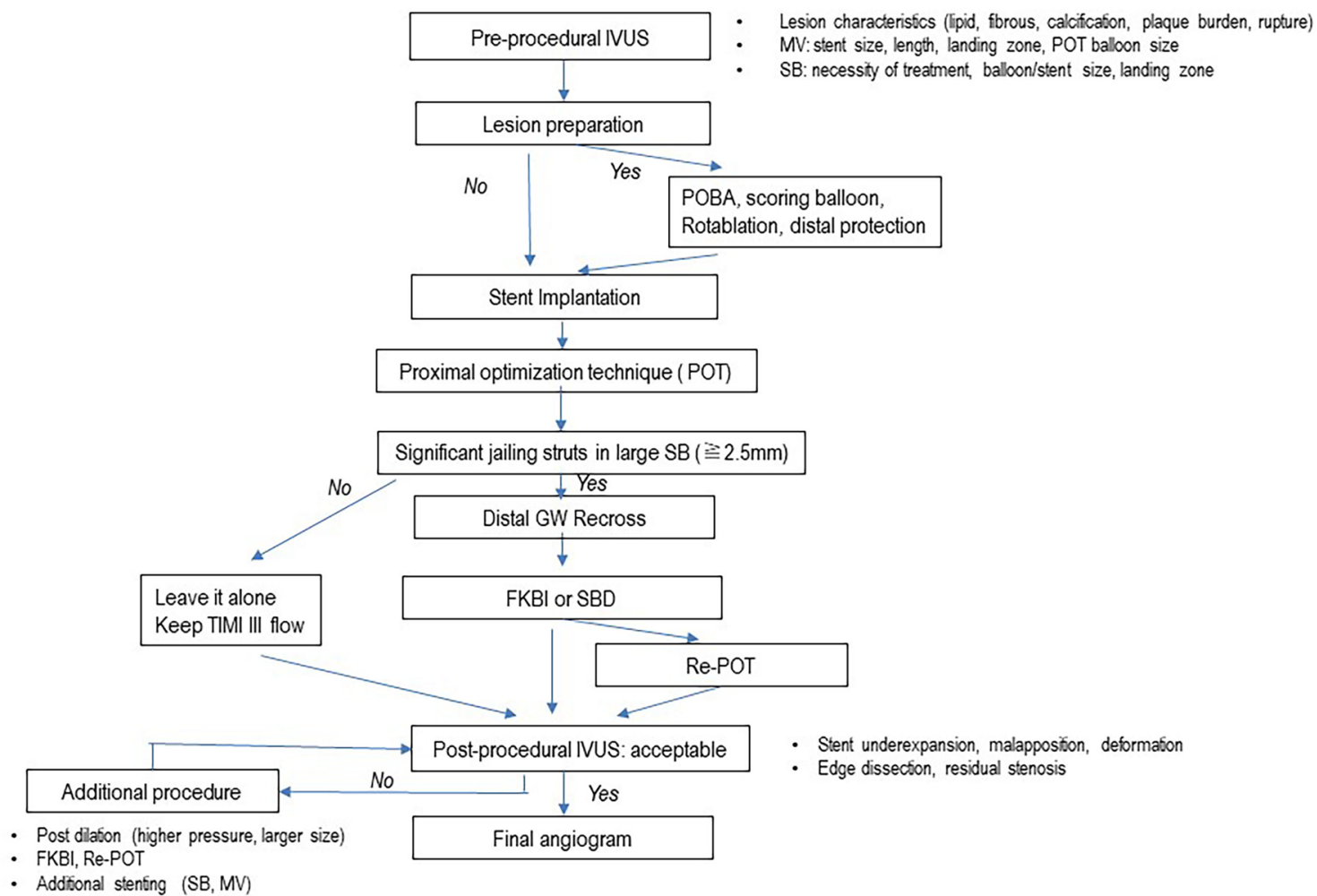

Fig. 4 Flow of IVUS-guided bifurcation PCI

is added as a more meticulous step. When suboptimal GW recrossing and SB ostial dilation or stent malapposition/deformation is found, additional optimization procedures should be considered.

\section{Summary}

The accurate morphological assessment of MV and SB using an intra-luminal imaging device before and after the procedure are important for optimization of the device during 


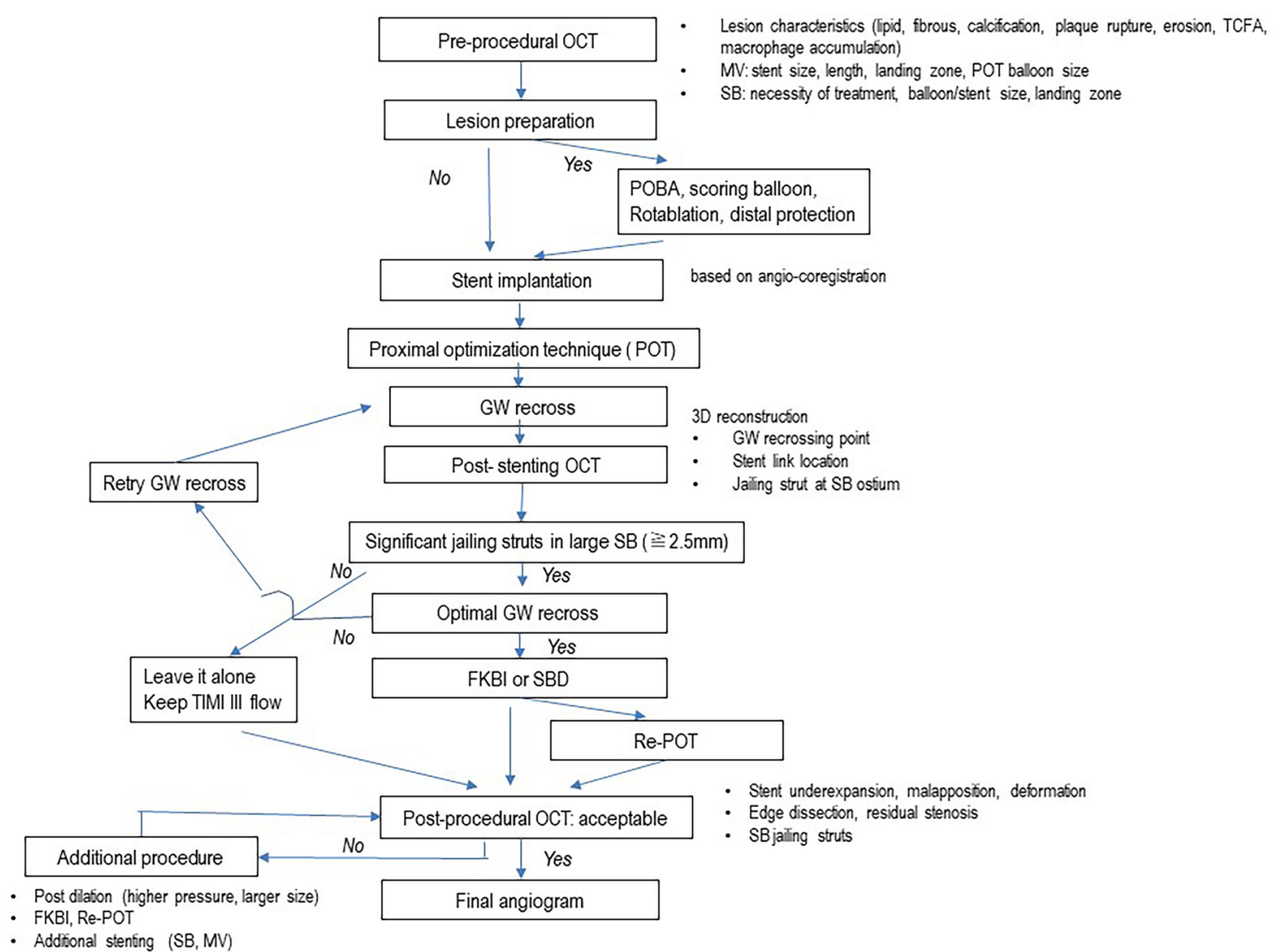

Fig. 5 Flow of OCT-guided bifurcation PCI

complex bifurcation PCI procedures. Imaging guidance can provide adequate stent expansion, less malapposition, appropriate stent landing, and treatment of dissection; which lead to improvement of clinical outcome. Moreover, the assessment of the recrossing position and stent link location with 3D- OCT imaging has the potential to improve the clinical outcome due to optimal SB treatment.

Open Access This article is licensed under a Creative Commons Attribution 4.0 International License, which permits use, sharing, adaptation, distribution and reproduction in any medium or format, as long as you give appropriate credit to the original author(s) and the source, provide a link to the Creative Commons licence, and indicate if changes were made. The images or other third party material in this article are included in the article's Creative Commons licence, unless indicated otherwise in a credit line to the material. If material is not included in the article's Creative Commons licence and your intended use is not permitted by statutory regulation or exceeds the permitted use, you will need to obtain permission directly from the copyright holder. To view a copy of this licence, visit http://creativecommons.org/licenses/by/4.0/.

\section{References}

1. Sawaya FJ, Lefèvre T, Chevalier B, Garot P, Hovasse T, Morice $\mathrm{MC}$, et al. Contemporary approach to coronary bifurcation lesion treatment. JACC Cardiovasc Interv. 2016;9:1861-78.
2. Tu S, Jing J, Holm NR, Onsea K, Zhang T, Adriaenssens T, et al. In vivo assessment of bifurcation optimal viewing angles and bifurcation angles by three-dimensional (3D) quantitative coronary angiography. Int J Cardiovasc Imaging. 2012;28:1617-25.

3. Tearney GJ, Regar E, Akasaka T, Adriaenssens T, Barlis P, Bezerra HG, et al. Consensus standards for acquisition, measurement, and reporting of intravascular optical coherence tomography studies: a report from the International Working Group for Intravascular Optical Coherence Tomography Standardization and Validation. J Am Coll Cardiol. 2012;59:1058-72.

4. Prati F, Guagliumi G, Mintz GS, Costa M, Regar E, Akasaka T et al. Expert's OCT Review Document. Expert review document part 2: methodology, terminology and clinical applications of optical coherence tomography for the assessment of interventional procedures. Eur Heart J. 2012;33:2513-20.

5. Kim JS, Hong MK, Ko YG, Choi D, Yoon JH, Choi SH, et al. Impact of intravascular ultrasound guidance on long-term clinical outcomes in patients treated with drug-eluting stent for bifurcation lesions: data from a Korean multicenter bifurcation registry. Am Heart J. 2011;161:180-7.

6. Raber L, Mintz GS, Koskinas KC, Johnson TW, Holm NR, Onuma $\mathrm{Y}$ et al. Clinical use of intracoronary imaging. Part 1: guidance and optimization of coronary interventions. An expert consensus document of the European Association of Percutaneous Cardiovascular Interventions. Eur Heart J 2018;39:3281-3300.

7. Hong SJ, Kim BK, Shin DH, Nam CM, Kim JS, Ko YG, et al. Effect of intravascular ultrasound-guided vs angiography-guided 
everolimus-eluting stent implantation: the IVUS-XPL randomized clinical trial. JAMA. 2015;314:2155-63.

8. Zhang J, Gao X, Kan J, Ge Z, Han L, Lu S, et al. Intravascular ultrasound versus angiography-guided drug-eluting stent implantation: the ULTIMATE trial. J Am Coll Cardiol. 2018;72:3126-37.

9. Zhang YJ, Pang S, Chen XY, Bourantas CV, Pan DR, Dong $\mathrm{SJ}$, et al. Comparison of intravascular ultrasound guided versus angiography guided drug eluting stent implantation: a systematic review and meta-analysis. BMC Cardiovasc Disord. 2015;15:153.

10. Park S-J, Kim Y-H, Park D-W, Lee SW, Kim WJ, Suh J, et al. Impact of intravascular ultrasound guidance on long-term mortality in stenting for unprotected left main coronary artery stenosis. Circ Cardiovasc Interv. 2009;2:167-77.

11. de la Torre Hernandez JM, Baz Alonso JA, Gomez Hospital JA, et al. Clinical impact of intravascular ultrasound guidance in drug-eluting stent implantation for unprotected left main coronary disease: pooled analysis at the patient-level of 4 registries. JACC Cardiovasc Interv. 2014;7:244-54.

12. Andell P, Karlsson S, Mohammad MA, Alfonso Manterola F, Garcia Camarero T, Gimeno de Carlos F et al. Intravascular ultrasound guidance is associated with better outcome in patients undergoing unprotected left main coronary artery stenting compared with angiography guidance alone. Circ Cardiovasc Interv 2017;10.

13. Gonzalo N, Garcia-Garcia HM, Regar E, Barlis P, Wentzel $\mathrm{J}$, Onuma $\mathrm{Y}$, et al. In vivo assessment of high-risk coronary plaques at bifurcations with combined intravascular ultrasound and optical coherence tomography. JACC Cardiovasc Imaging. 2009;2:473-82.

14. Badak O, Schoenhagen P, Tsunoda T, Magyar WA, Coughlin J, Kapadia S, et al. Characteristics of atherosclerotic plaque distribution in coronary artery bifurcations: an intravascular ultrasound analysis. Coron Artery Dis. 2003;14:309-16.

15. Kim YH, Lee JH, Roh JH, Ahn JM, Yoon SH, Park DW, et al. Randomized comparisons between different stenting approaches for bifurcation coronary lesions with or without side branch stenosis. JACC Cardiovasc Interv. 2015;8:550-60.

16. Neumann FJ, Sousa-Uva M, Ahlsson A, Alfonso F, Banning AP, Benedetto U, et al. ESC/EACTS Guidelines on myocardial revascularization. EuroIntervention. 2018;2019(14):1435-534.

17. Frick K, Michael TT, Alomar M, Mohammed A, Rangan BV, Abdullah S, et al. Low molecular weight dextran provides similar optical coherence tomography coronary imaging compared to radiographic contrast media. Catheter Cardiovasc Interv. 2014;84:727-31.

18. Ozaki Y, Kitabata H, Tsujioka H, Hosokawa S, Kashiwagi $\mathrm{M}$, Ishibashi $\mathrm{K}$, et al. Comparison of contrast media and lowmolecular-weight dextran for frequency-domain optical coherence tomography. Circ J. 2012;76:922-7.

19. Kubo T, Akasaka T, Shite J, Suzuki T, Uemura S, Yu B at al. OCT compared with IVUS in a coronary lesion assessment: the OPUS-CLASS study. JACC Cardiovasc Imaging. 2013;6:1095-1104.

20. Liu Y, Shimamura K, Kubo T, Tanaka A, Kitabata H, Ino Y, et al. Comparison of longitudinal geometric measurement in human coronary arteries between frequency-domain optical coherence tomography and intravascular ultrasound. Int J Cardiovasc Imaging. 2014;30:271-7.

21. Tanaka A, Imanishi T, Kitabata H, Kubo T, Takarada S, Tanimoto $\mathrm{T}$, et al. Lipid-rich plaque and myocardial perfusion after successful stenting in patients with non-ST-segment elevation acute coronary syndrome: an optical coherence tomography study. Eur Heart J. 2009;30:1348-55.
22. Gonzalo N, Serruys PW, Okamura T, Shen ZJ, Garcia-Garcia HM, Onuma Y, et al. Relation between plaque type and dissections at the edges after stent implantation: an optical coherence tomography study. Int J Cardiol. 2011;150:151-5.

23. Murasato Y, Mori T, Okamura T, Nagoshi R, Fujimura T, Yamawaki M, et al. Efficacy of the proximal optimization technique on crossover stenting in coronary bifurcation lesions in the 3D-OCT bifurcation registry. Int J Cardiovasc Imaging. 2019;35:981-90.

24. Finet G, Derimay F, Motreff P, Guerin P, Pilet P, Ohayon J, et al. Comparative analysis of sequential proximal optimizing technique versus kissing balloon inflation technique in provisional bifurcation stenting: fractal coronary bifurcation bench test. JACC Cardiovasc Interv. 2015;8:1308-17.

25. Alegria-Barrero E, Foin N, Chan PH, Syrseloudis D, Lindsay AC, Dimopolous K, et al. Optical coherence tomography for guidance of distal cell recrossing in bifurcation stenting: choosing the right cell matters. EuroIntervention. 2012;8:205-13.

26. Okamura T, Onuma Y, Yamada J, Iqbal J, Tateishi H, Nao T, et al. 3D optical coherence tomography: new insights into the process of optimal rewiring of side branches during bifurcational stenting. EuroIntervention. 2014;10:907-15.

27. Okamura T, Nagoshi R, Fujimura T, Murasato Y, Yamawaki $\mathrm{M}$, Ono S, et al. Impact of guidewire recrossing point into stent jailed side branch for optimal kissing balloon dilatation: core lab 3D optical coherence tomography analysis. EuroIntervention. 2018;13:e1785-e17931793.

28. Nagoshi R, Okamura T, Murasato Y, Fujimura T, Yamawaki $\mathrm{M}$, Ono S, et al. Feasibility and usefulness of three-dimensional optical coherence tomography guidance for optimal side branch treatment in coronary bifurcation stenting. Int J Cardiol. 2018;250:270-4.

29. Ali ZA, Maehara A, Genereux P, Shlofmitz RA, Fabbiocchi F, Nazif TM, et al. Optical coherence tomography compared with intravascular ultrasound and with angiography to guide coronary stent implantation (ILUMIEN III: OPTIMIZE PCI): a randomised controlled trial. Lancet. 2016;388:2618-28.

30. Kubo T, Shinke T, Okamura T, Hibi K, Nakazawa G, Morino $\mathrm{Y}$ et al. Optical frequency domain imaging vs. intravascular ultrasound in percutaneous coronary intervention (OPINION trial): one-year angiographic and clinical results. Eur Heart J. 2017;38:3139-3147.

31. Maehara A, Ben-Yehuda O, Ali Z, Wijns W, Bezerra HG, Shite $\mathrm{J}$, et al. Comparison of Stent expansion guided by optical coherence tomography versus intravascular ultrasound: the ILUMIEN II study (Observational Study of Optical Coherence Tomography $[\mathrm{OCT}]$ in Patients Undergoing Fractional Flow Reserve [FFR] and Percutaneous Coronary Intervention). JACC Cardiovasc Interv. 2015;8:1704-14.

32. Kobayashi Y, Okura H, Kume T, Yamada R, Kobayashi Y, Fukuhara $\mathrm{K}$, et al. Impact of target lesion coronary calcification on stent expansion. Circ J. 2014;78:2209-14.

33. Fujii K, Carlier SG, Mintz GS, Yang YM, Moussa I, Weisz G, et al. Stent underexpansion and residual reference segment stenosis are related to stent thrombosis after sirolimus-eluting stent implantation: an intravascular ultrasound study. J Am Coll Cardiol. 2005;45:995-8.

34. Hong M-K, Mintz GS, Lee CW, Park DW, Choi BR, Park KH, et al. Intravascular ultrasound predictors of angiographic restenosis after sirolimus-eluting stent implantation. Eur Heart J. 2006;27:1305-10.

35. Doi H, Maehara A, Mintz GS, Yu A, Wang H, Mandinov L et al. Impact of post-intervention minimal stent area on 9-month followup patency of paclitaxel-eluting stents: an integrated intravascular ultrasound analysis from the TAXUS IV, V, and VI and TAXUS 
ATLAS Workhorse, Long Lesion, and Direct Stent Trials. JACC Cardiovasc Interv 2009;2:1269-75.

36. Sonoda S, Morino Y, Ako J, Terashima M, Hassan AH, Bonneau $\mathrm{HN}$, et al. Impact of final stent dimensions on long-term results following sirolimus-eluting stent implantation: serial intravascular ultrasound analysis from the sirius trial. J Am Coll Cardiol. 2004;43:1959-63.

37. Song HG, Kang SJ, Ahn JM, Kim WJ, Lee JY, Park DW, et al. Intravascular ultrasound assessment of optimal stent area to prevent in-stent restenosis after zotarolimus-, everolimus-, and sirolimus-eluting stent implantation. Catheter Cardiovasc Interv. 2014;83:873-8.

38. Kang S-J, Ahn J-M, Song H Kim WJ, Lee JY, Park DW, et al. Comprehensive intravascular ultrasound assessment of stent area and its impact on restenosis and adverse cardiac events in 403 patients with unprotected left main disease. Circ Cardiovasc Interv. 2011;4:562-569.

39. Morino Y, Tamiya S, Masuda N, Kawamura Y, Nagaoka M, Matsukage $\mathrm{T}$, et al. Intravascular ultrasound criteria for determination of optimal longitudinal positioning of sirolimus-eluting stents. Circ J. 2010;74:1609-16.

40. Kang SJ, Cho YR, Park GM, Ahn JM, Kim WJ, Lee JY, et al. Intravascular ultrasound predictors for edge restenosis after newer generation drug-eluting stent implantation. Am J Cardiol. 2013;111:1408-14.

41. Soeda T, Uemura S, Park SJ, Jang Y, Lee S, Cho JM, et al. Incidence and clinical significance of poststent optical coherence tomography findings: one-year follow-up study from a multicenter registry. Circulation. 2015;132:1020-9.

42. Huo Y, Finet G, Lefevre T, Louvard Y, Moussa I, Kassab GS. Which diameter and angle rule provides optimal flow patterns in a coronary bifurcation? J Biomech. 2012;45:1273-9.

43. Nakamura D, Wijns W, Price MJ, Jones MR, Barbato E, Akasaka T, et al. New volumetric analysis method for stent expansion and its correlation with final fractional flow reserve and clinical outcome: an ILUMIEN I Substudy. JACC Cardiovasc Interv. 2018;11:1467-78.

44. Foin N. Final proximal post-dilatation is necessary after kissing balloon in bifurcation stenting. EuroIntervention. 2011;7:597-604

45. Ojeda S, Pan M, Martín P, Mazuelos F, Suárez de Lezo J, Romero $\mathrm{M}$ et al. Immediate results and long-term clinical outcome of patients with unprotected distal left main restenosis: the CORPAL Registry (Córdoba and Las Palmas). JACC: Cardiovascular Interventions 2014;7:212-221.

46. Oviedo C, Maehara A, Mintz GS, Araki H, Choi SY, Tsujita K, et al. Intravascular ultrasound classification of plaque distribution in left main coronary artery bifurcations: where is the plaque really located? Circ Cardiovasc Interv. 2010;3:105-12.

47. Sakurai R, Ako J, Morino Y, Sonoda S, Kaneda H, Terashima M, et al. Predictors of edge stenosis following sirolimus-eluting stent deployment (a quantitative intravascular ultrasound analysis from the SIRIUS trial). Am J Cardiol. 2005;96:1251-3.

48. Takagi K, Fujino Y, Naganuma T, Watanabe Y, Yabushita H, Mitomo S, et al. Impact of a combination of full coverage stenting and proximal optimization technique on long term outcome for unprotected distal left main disease. Cardiovasc Revasc Med. 2016;17:515-21.

49. Steigen TK, Maeng M, Wiseth R, Erglis A, Kumsars I, Narbute I, et al. Randomized study on simple versus complex stenting of coronary artery bifurcation lesions: the Nordic bifurcation study. Circulation. 2006;114:1955-61.

50. Hildick-Smith D, de Belder AJ, Cooter N, Curzen NP, Clayton TC, Oldroyd KG, et al. Randomized trial of simple versus complex drug-eluting stenting for bifurcation lesions: the British
Bifurcation Coronary Study: old, new, and evolving strategies. Circulation. 2010;121:1235-43.

51. Chen SL, Zhang JJ, Han Y, Kan J, Chen L, Qiu C, et al. Double kissing crush versus provisional stenting for left main distal bifurcation lesions: DKCRUSH-V Randomized Trial. J Am Coll Cardiol. 2017;70:2605-17.

52. Murasato Y, Kinoshita Y, Yamawaki M, Shinke T, Otake H, Takeda Y, et al. Efficacy of kissing balloon inflation after provisional stenting in bifurcation lesions guided by intravascular ultrasound: short and midterm results of the J-REVERSE registry. EuroIntervention. 2016;11:e1237-e12481248.

53. Cheneau E, Leborgne L, Mintz GS, Kotani J, Pichard AD, Satler LF, et al. Predictors of subacute stent thrombosis: results of a systematic intravascular ultrasound study. Circulation. 2003;108:43-7.

54. Yu CW, Yang JH, Song YB, Hahn JY, Choi SH, Choi JH, et al. Long-term clinical outcomes of final kissing ballooning in coronary bifurcation lesions treated with the 1-stent technique: results from the COBIS II Registry (Korean Coronary Bifurcation Stenting Registry). JACC Cardiovasc Interv. 2015;8:1297-307.

55. Gwon HC, Hahn JY, Koo BK, Song YB, Choi SH, Choi JH, et al. Final kissing ballooning and long-term clinical outcomes in coronary bifurcation lesions treated with 1-stent technique: results from the COBIS registry. Heart. 2012;98:225-31.

56. Niemela M, Kervinen K, Erglis A, Holm NR, Maeng M, Christiansen EH, et al. Randomized comparison of final kissing balloon dilatation versus no final kissing balloon dilatation in patients with coronary bifurcation lesions treated with main vessel stenting: the Nordic-Baltic Bifurcation Study III. Circulation. 2011;123:79-86.

57. Rahman S, Leesar T, Cilingiroglu M, Effat M, Arif I, Helmy T, et al. Impact of kissing balloon inflation on the main vessel stent volume, area, and symmetry after side-branch dilation in patients with coronary bifurcation lesions: a serial volumetric intravascular ultrasound study. JACC Cardiovasc Interv. 2013;6:923-31.

58. Guerin P, Pilet P, Finet G, Gouëffic Y, N'Guyen JM, Crochet $\mathrm{D}$, et al. Drug-eluting stents in bifurcations: bench study of strut deformation and coating lesions. Circ Cardiovasc Interv. 2010;3:120-6.

59. Murasato Y, Iwasaki K, Yamamoto T, Yagi T, Hikichi Y, Suematsu Y, et al. Optimal kissing balloon inflation after single-stent deployment in a coronary bifurcation model. EuroIntervention. 2014;10:934-41.

60. Takahashi H, Otake H, Shinke T, Murasato Y, Kinoshita Y, Yamawaki $\mathrm{M}$, et al. Impact of final kissing balloon inflation on vessel healing following drug-eluting stent implantation: Insight from the optical coherence tomography sub-study of the J-REVERSE trial. J Cardiol. 2016;68:504-11.

61. Alfonso F, Hernández C, Pérez-Vizcayno MaJ, Hernández R, Fernández-Ortíz A, Escaned J et al. Fate of stent-related side branches after coronary intervention in patients with in-stent restenosis. J Am Coll Cardiol. 2000;36:1549-1556.

62. Gil RJ, Vassilev D, Formuszewicz R, Rusicka-Piekarz T, Doganov A. The carina angle-new geometrical parameter associated with periprocedural side branch compromise and the long-term results in coronary bifurcation lesions with main vessel stenting only. $\mathrm{J}$ Interv Cardiol. 2009;22:E1-E10.

63. Hahn JY, Chun WJ, Kim JH, Song YB, Oh JH, Koo BK, et al. Predictors and outcomes of side branch occlusion after main vessel stenting in coronary bifurcation lesions: results from the COBIS II registry (COronary BIfurcation Stenting). J Am Coll Cardiol. 2013;62:1654-9.

64. Furukawa E, Hibi K, Kosuge M, Nakatogawa T, Toda N, Takamura $\mathrm{T}$, et al. Intravascular ultrasound predictors of side branch occlusion in bifurcation lesions after percutaneous coronary intervention. Circ J. 2005;69:325-30. 
65. Vassilev D, Gil RJ, Koo BK, Gibson CM, Nguyen T, Hoang T, et al. The determinants of side branch compromise after main vessel stenting in coronary bifurcation lesions. Kardiol Pol. 2012;70:989-97.

66. Yamawaki M, Murasato Y, Kinoshita Y, Fujii K, Fujino Y, Shinke $\mathrm{T}$, et al. Mechanism of Residual Lumen Stenosis at the Side Branch Ostium After Final Kissing Balloon Inflation: A Volumetric Intracoronary Ultrasound Study of Coronary Bifurcation Lesions. J Interv Cardiol. 2016;29:188-96.

67. Xu J, Hahn JY, Song YB, Choi SH, Choi JH, Lu C, et al. Carina shift versus plaque shift for aggravation of side branch ostial stenosis in bifurcation lesions: volumetric intravascular ultrasound analysis of both branches. Circ Cardiovasc Interv. 2012;5:657-62.

68. Costa RA, Feres F, Staico R, Abizaid A, Costa JR Jr, Siqueira D, et al. Vessel remodeling and plaque distribution in side branch of complex coronary bifurcation lesions: a grayscale intravascular ultrasound study. Int J Cardiovasc Imaging. 2013;29:1657-66.

69. Medina A, Martin P, Suarez de Lezo J, Amador C, Suárez de Lezo J, Pan M et al. Vulnerable carina anatomy and ostial lesions in the left anterior descending coronary artery after floating-stent treatment. Rev Esp Cardiol. 2009;62:1240-9.

70. Sato K, Naganuma T, Costopoulos C, Takebayashi H, Goto K, Miyazaki T, et al. Calcification analysis by intravascular ultrasound to define a predictor of left circumflex narrowing after cross-over stenting for unprotected left main bifurcation lesions. Cardiovasc Revasc Med. 2014;15:80-5.

71. Fujino Y, Attizzani GF, Tahara S, Takagi K, Naganuma T, Wang $\mathrm{W}$, et al. Impact of main-branch calcified plaque on side-branch stenosis in bifurcation stenting: an optical coherence tomography study. Int J Cardiol. 2014; 176:1056-60.

72. Watanabe M, Uemura S, Sugawara Y, Ueda T, Soeda T, Takeda $\mathrm{Y}$, et al. Side branch complication after a single-stent crossover technique: prediction with frequency domain optical coherence tomography. Coron Artery Dis. 2014;25:321-9.

73. Farooq V, Serruys PW, Heo JH, Gogas BD, Okamura T, GomezLara $\mathbf{J}$, et al. New insights into the coronary artery bifurcation hypothesis-generating concepts utilizing 3-dimensional optical frequency domain imaging. JACC Cardiovasc Interv. 2011;4:921-31.

74. Foin N, Torii R, Alegria E, Sen S, Petraco R, Nijjer S, et al. Location of side branch access critically affects results in bifurcation stenting: Insights from bench modeling and computational flow simulation. Int J Cardiol. 2013;168:3623-8.

75. Nagoshi R, Okamura T, Shite J. A Novel Push-Fold Method for Removing Side Branch-Jailed Stent Struts Under 3D Optical
Coherence Tomography Guidance. JACC Cardiovasc Interv. 2016;9:e107-e109109.

76. Fujino Y, Attizzani GF, Tahara S, Naganuma T, Takagi K, Yabushita $\mathrm{H}$, et al. Difference in vascular response between sirolimus-eluting- and everolimus-eluting stents in ostial left circumflex artery after unprotected left main as observed by optical coherence tomography. Int J Cardiol. 2017;230:284-92.

77. Mori H, Torii S, Harari E, Jinnouchi H, Brauman R, Smith S, et al. Pathological mechanisms of left main stent failure. Int J Cardiol. 2018;263:9-16.

78. Gao XF, Kan J, Zhang YJ, Zhang JJ, Tian NL, Ye F, et al. Comparison of one-year clinical outcomes between intravascular ultrasound-guided versus angiography-guided implantation of drugeluting stents for left main lesions: a single-center analysis of a 1,016-patient cohort. Patient Prefer Adherence. 2014;8:1299-309.

79. Tan Q, Wang Q, Liu D, Zhang S, Zhang Y, Li Y. Intravascular ultrasound-guided unprotected left main coronary artery stenting in the elderly. Saudi Med J. 2015;36:549-53.

80. Kim SH, Kim YH, Kang SJ, Park DW, Lee SW, Lee CW, et al. Long-term outcomes of intravascular ultrasound-guided stenting in coronary bifurcation lesions. Am J Cardiol. 2010;106:612-8.

81. Chen SL, Ye F, Zhang JJ, Tian NL, Liu ZZ, Santoso T, et al. Intravascular ultrasound-guided systematic two-stent techniques for coronary bifurcation lesions and reduced late stent thrombosis. Catheter Cardiovasc Interv. 2013;81:456-63.

82. Kini AS, Vengrenyuk Y, Pena J, Yoshimura T, Panwar SR, Motoyama S, et al. Plaque morphology predictors of side branch occlusion after provisional stenting in coronary bifurcation lesion: Results of optical coherence tomography bifurcation study (ORBID). Catheter Cardiovasc Interv. 2017;89:259-68.

83. Kume T, Yamada R, Terumasa K, Tamada T, Imai K, Fukuhara $\mathrm{K}$, et al. Neointimal coverage of jailed side branches in coronary bifurcation lesions: an optical coherence tomography analysis. Coron Artery Dis. 2018;29:114-8.

84. Fujimura T, Okamura T, Tateishi H, Nakamura T, Yamada J, Oda $\mathrm{T}$, et al. Serial changes in the side-branch ostial area after mainvessel stenting with kissing balloon inflation for coronary bifurcation lesions, assessed by 3D optical coherence tomography. Eur Heart J Cardiovasc Imaging. 2018;19:1117-25.

Publisher's Note Springer Nature remains neutral with regard to jurisdictional claims in published maps and institutional affiliations. 